\title{
Stent placement above the sphincter of Oddi permits implementation of neoadjuvant chemotherapy in patients with initially unresectable Klatskin tumor
}

Authors

Institutions
Kensuke Kubota' ${ }^{1}$, Sho Hasegawa', Akito Iwasaki ${ }^{1}$, Takamitsu Sato ${ }^{1}$, Yuji Fujita', Kunihiro Hosono', Atsushi Nakajima', Ryutaro Mori², Ryusei Matsuyama ${ }^{2}$, Itaru Endo ${ }^{2}$

Gastroenterology and Hepatology, Yokohama City University, Yokohama, Japan

${ }_{2}^{2}$ Gastroenterological Surgery, Yokohama City University, Yokohama, Japan submitted 28. January 2016 accepted after revision 29. January 2016

\section{Bibliography}

Dol http://dx.doi.org/ 10.1055/s-0042-102246 Published online: 30.3.2016 Endoscopy International Open 2016; 04: E427-E433

(c) Georg Thieme Verlag KG Stuttgart · New York E-ISSN 2196-9736

\section{Corresponding author} Kensuke Kubota

3-9 Fukuura

Kanazawa

Yokohama

Japan 2360004

Fax: +045-784-3546

kubotak@yokohama-cu.ac.jp
Background and study aims: Neoadjuvant chemotherapy (NAC) may lead to a successful marginnegative resection in patients with initially unresectable locally advanced Klatskin tumor (IULAKT). Use of removable plastic stents is preferable for the safe implementation of NAC in patients with IULAKT to reduce the risk of recurrent cholangitis. Our aim was to evaluate the efficacy associated with the use of plastic stents placed across the stenosis and above the papilla (above stent) during NAC.

Patients and methods: In this study, we stratified the patients into two groups chronologically with respect to the period of stent placement: above stent group $(\mathrm{n}=17)$ and across stent group $(\mathrm{n}=$ 23) (plastic stent across the sphincter of Oddi). Results: The median stent patency period was 99 days in the above stent group and 31 days in the

\section{Introduction}

\section{$\nabla$}

Neoadjuvant chemotherapy (NAC) followed by surgical resection in patients with Klatskin tumor and hilar cholangiocarcinoma may be promising for subsequent margin-negative surgical resection (R0). Although the prognosis of Klatskin tumor is poor, 5-year survival after R0 resection of Klatskin tumor is in the range of $30 \%$ to $50 \%$ [1]. There is no general consensus when it comes to using stents for Klatskin tumor during NAC. There are numerous papers regarding the placing of plastic stents across the stenosis and above the papilla (above stent) for biliary tract cancer. However, these data comprise studies assessing lesions at multiple locations of the bile duct, such as the hilar part, gallbladder, and pancreatic head [2]. Bile duct cancer comprises four heterogeneous tumor types affecting the hilar and upper part of the bile duct, gallbladder, lower part of the bile duct, and the ampulla of Vater. Prognosis differs according to the location of the tumor. Klatskin tumor, originally arising from the hilar across stent group $(P<0.0001)$. The number of stents $(P=0.017)$ and the rate of emerging undrained cholangitis areas $(P=0.025)$ were significantly reduced in the above stent group than the counterpart. Regarding time to recurrent biliary obstruction, the above stent group had a longer duration than the across stent group (log rank test, $P=0.004$ ). Length of hospital stay was significantly shorter for the above stent group than the across stent group $(P=0.0475)$. Multivariate analysis revealed that above stent placement (odds ratio $=33.638, P=0.0048$ ) was significantly associated with stent patency over a period of 90 days. Conclusions: Above stent placement should be considered for the relief of biliary obstruction and potentially reduces the cost for patients with IULAKT scheduled to receive NAC.

part of the bile duct, has the worst 5-year-survival prognosis after resection of all of the biliary tract cancers [3] and in most patients, tumors are indicated as inoperable by the time of diagnosis [4]. Here we attempt to shed light on unmixed Klatskin tumors during NAC by assessing the use of plastic stent placement across the stenosis and above the papilla. NAC has recently been shown to provide patients whose tumors were initially unresectable with a chance of R0 resection [5]; down-staging of chemotherapy for patients with unresectable Klatskin tumor has also been attempted [6]. Patients with Klatskin tumor presenting with symptomatic obstructive cholangitis required placement of a biliary stent throughout a safe and effective NAC period. Across stent (plastic stent across the sphincter of Oddi) placement is a choice for symptomatic patients with resectable Klatskin tumor. However, across stent can induce reflux cholangitis [7] and multiple across stents are required for endoscopic sphincterotomy. Conversely, endoscopic nasal biliary drainage (ENBD) is advantageous because it reduces 
the rate of reflux cholangitis [8] in patients who have to endure it for a period of 2 or 3 weeks. Endoscopic metal stents are not suitable for surgery because the surgical margin attached with them obscures identification. Above stent placement is promising because it can reduce cholangitis reflux $[9,10]$. This is the first study comparing above stent and across stent placements coupled with ongoing NAC for unmixed Klatskin tumors. NAC may lead to a successful $\mathrm{R} 0$ resection in patients with initially unresectable locally advanced Klatskin tumor (IULAKT). NAC over 3 months ( $>90$ days) to control biliary infection and jaundice is essential for safe NAC with minimal interruption. Above stent placement could be an alternative to the conventional method because of its prophylactic effect against stent dysfunction, wherein occlusion of stent or reflux of duodenal juice is a consequence. Our aim was to evaluate the feasibility, efficacy, and safety associated with using above stent placement compared with those associated with using across stent placement during NAC for unmixed Klatskin tumors. This study can be a precursor to RCT of preoperative stent placement for IULAKT.

\section{Patients and methods}

\section{Study design}

Patients with IULAKT were retrospectively enrolled at a single, tertiary, pancreaticobiliary referral center (Yokohama City University Hospital, Yokohama, Japan). We studied unmixed Klatskin tumors, excluding gallbladder cancer, intrahepatic cholangiocarcinoma, and biliary cancer arising from pancreatic head lesions and metastatic Klatskin tumors. We hypothesized that in comparison with across stent placement, above stent placement may result in a longer period of time to recurrent biliary obstruction (TRBO) based on the 2014 Tokyo criteria for transpapillary biliary stenting [11] and could contribute to a better prognosis without complications. The first end point was to assess the TRBO of above stent placement and determine whether it is longer than the conventional method (across stent placement). The second end point was to evaluate the amelioration of prognosis using above stent placement compared with that using across stent placement. The final end point was to study favorable factors for stent patency lasting more than 90 days, which was the minimum period required to complete NAC for Klatskin tumor. We reviewed the outcomes of consecutive patients with IULAKT, who presented with symptomatic biliary obstruction, and stratified the patients chronologically according to the period of stent placement into two groups: the above stent and across stent placement groups. The latter included a conventional plastic stent placed across the sphincter of Oddi (across stent), and the study was conducted between January 2012 and July 2015.

\section{Case definition of IULAKT}

Klatskin tumor, which was limited to the P point (the bifurcation of the anterior and posterior branches of the portal hepatic vein) or the $U$ point (the bend between the transverse and umbilical portions of the left portal vein), was regarded as resectable based on preoperative three-dimensional (3-D) images [12]. Furthermore, a tumor without distant and/or para aorta lymph node metastases was regarded as resectable. Patients with initially unresectable disease were candidates for NAC. NAC indications were as follows: 1. tumor extending beyond both the $P$ and $U$ points [12]; 2. Extensive hepatic artery or main trunk of the portal vein involvements that would not be amenable to reconstruction; and
3. Presence of perihilar lymph node metastasis. The diagnosis was made on the basis of the cholangiography findings and 3-D images using multi-detector computed tomography (MDCT) [12]. Klatskin tumor showed nodular or sclerosing types on imaging diagnosis and histopathology. However, the sclerosing type showed annular thickening of the bile duct with longitudinal and radical tumor infiltration. It is often hard to evaluate the tumor size [13] based on RECIST [14]. In such situations, we regarded stable disease (SD) as that with no recognized new metastasis or tumor invasion. Tissue assessment by endoscopic biopsy was also pivotal to the diagnosis of IULAKT. The Yokohama City University Hospital review board approved this study (B141201004). Written informed consent was obtained from all enrolled patients.

\section{Endoscopy, guidewires, and stents}

Experienced endoscopists (those who performed more than 200 endoscopies per year) conducted stent placement using a videoscope (JF260V; Olympus Medical systems, Tokyo, Japan). Guidewires (0.025 Visigride, Olympus Medical systems, Tokyo, Japan or 0.035 Jagwire, Microvasive Endoscopy, Boston Scientific Corp, Natick, MA, USA) were used as a seeking guide. The above stent device was a $7 \mathrm{~F}$ straight stent with one or two flaps at the hilar part (Cook Medical Inc, Bloomington, MN, USA). This device was tied with a nylon thread through the side hole at the anal site. We used a $7 \mathrm{~F}$ straight across stent device (Flexima, Microvasive Endoscopy, Boston scientific Corp, Natick, MA, USA). Endoscopic sphincterotomy (EST) was performed in cases where multiple across stent placements were required. We placed the above stent device(s) without EST.

\section{ENBD, stent placements, and chemotherapy}

First, ENBD was used to relieve cholangitis until the total bilirubin level was reduced below $2 \mathrm{mg} / \mathrm{dL}$. Then, ENBD was exchanged for above stent or across stent placement, immediately after cholangitis ceased. For stent placement toward resection, using a unilateral stent was planned to preserve the lobule on the future remnant hepatic lobe and was considered essential ( $\bullet$ Fig. 1 and - Fig.2). A bilateral stent placement ( $\bullet$ Fig. 3 and $\bullet$ Fig.4) was attempted if the unilateral stent placement was considered insufficient in controlling obstructive cholangitis or if cholangitis developed in the undrained hepatic lobe. The above stent device was attached to a string for its smooth retrieval [15] and placed between the proximal and distal sites of the stenosis and/or obstruction before being placed across the stricture without sphincterotomy. Then, one or two flaps of the hilar part were used to prevent dislocation. We used biopsy forceps by grasping the string or the flap and/or a stone retrieval balloon catheter to remove it from the hilar part of the bile duct. A gemcitabine (GEM)based regimen was used for NAC. This regimen provides patients with GEM at $1000 \mathrm{mg} / \mathrm{m}^{2}$ on day 1 through day 8 plus S- 160 , or $80 \mathrm{mg} /$ day on day 1 through day 14 of a 21-day cycle. After more than two or three courses of NAC, the resectability was evaluated based on RECIST [13]. It took more than 90 days to conduct and evaluate NAC in this study. If distant metastasis and/or newly formed lymph nodes were not recognized after NAC, we regarded it as SD. We attempted resection if the lesion indicated a partial response or if an SD was observed after two or three courses of NAC. Down-stage chemotherapy was continued until a maximum of 12 courses if we still regarded the patient unresectable after 3 months of chemotherapy. 


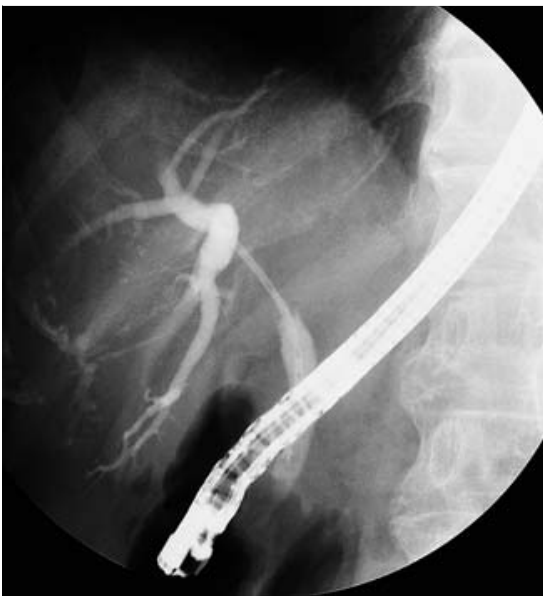

Fig. 1 An above stent was placed in the right lobe planned for left hepatectomy.

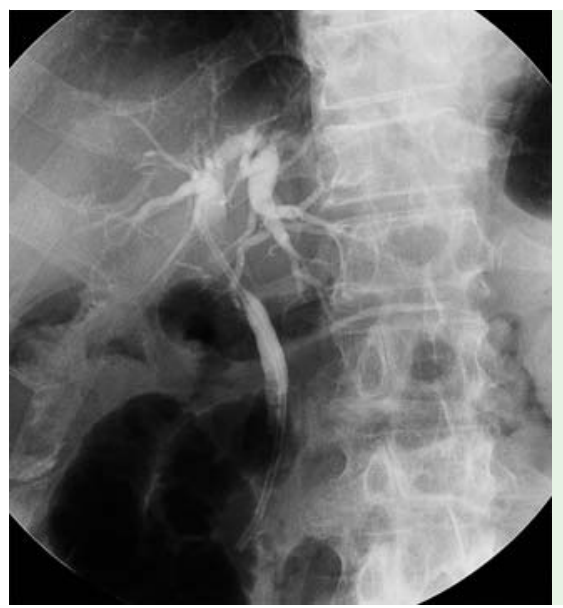

Fig. 2 An across stent across the ampulla was placed in the left lobe planned for right hepatectomy.

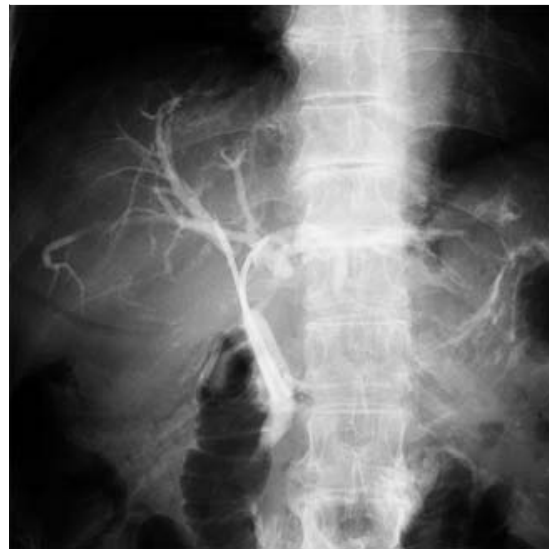

Fig.3 Double above stents were placed for right hepatectomy.

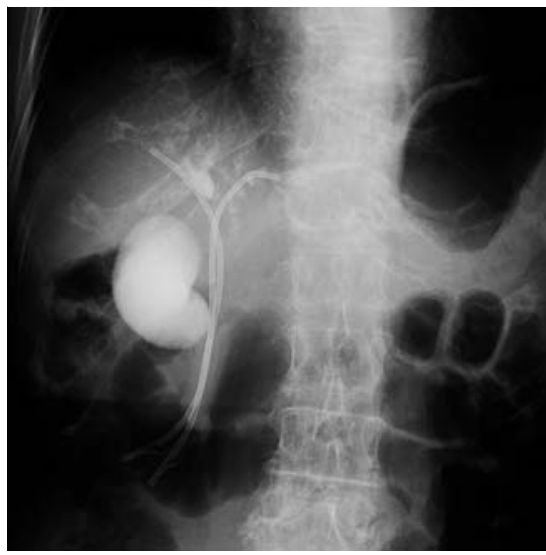

Fig. 4 Double across stents were placed for right hepatectomy. These stents ends were exposed to the duodenum.

\section{Reintervention study}

The above stent device was retrieved using forceps by grasping the string attached to it. Successful stent placement was defined as that in which serum total bilirubin level remained normal $(<1.2 \mathrm{mg} / \mathrm{dL})$ until 1 week after stent placement. We studied stent patency and prognosis. Stent dislocation comprised proximal and distal migration; proximal migration was defined as that located above the original stricture site and distal migration was defined as that located below the original stricture or where the stent had radiographically vanished [11]. Reflux cholangitis was defined as cholangitis because of the influx of duodenal contents, which impeded bile duct flow. This condition can cause immediate stent occlusion. Regarding the undrained area of cholangitis, we initially placed stents in the future remnant hepatic lobe. However, it was possible that undrained areas of cholangitis developed if we conducted drainage for additional lobes for the control of cholangitis during NAC. The time of emergency endoscopic retrograde cholangiopancreatography (ERCP) was indicated when a patient developed acute cholangitis or jaundice because of a drained/undrained hepatic lobe after stent placement [16]. The stent patency period was determined by the interval between stent placement and occlusion or the day of the surgery. Data on patient demographics, the rate of post-procedure pancreatitis [17], TRBO [11], surgical time, operative blood loss, length of hospital stay, complications after resection, the rate of R0 resection, and prognosis were studied.

\section{Statistical evaluation}

Data were analyzed using Stat View program (SAS Institute, Cary, NC, USA). Normality of distribution for categorical variables was compared using the $X^{2}$ test. Normality of distribution for continuous variables was analyzed using the Student's $t$-test. Non-normally distributed data were compared using the Mann - Whitney $\mathrm{U}$ test. Differences in categorical variables were tested using the Fisher's exact test. The primary outcome was TRBO and the secondary outcome was overall survival. TRBO and progression-free survival were analyzed using Kaplan-Meier curves and the log rank test based on the 2014 Tokyo criteria for transpapillary biliary stenting [11]. $P$ values of 0.05 or less were considered statistically significant. Multiple analyses were conducted using multiple regression analysis to identify factors associated with stent patency over 90 days. Candidate variables with $P$ values for association that were $<0.1$ on univariate analysis were considered as potential risk factors in multiple logistic regression analysis. We included all relevant patient demographics (age, sex, and serum CA19-9 level), tumor variables (tumor type [18], Bismuth type [19], artery invasion, hilar lymph node metastasis, and UICC stage), and stent variables (emergency ERCP, stent occlusion, stent migration, reflux cholangitis, and undrained area of cholangitis) in the multivariable analysis. Adjusted Hazards and 95\% confidence intervals (CIs) were reported. The significance level for all tests was two sided at $5 \%$.

\section{Results \\ V}

\section{Patient demographics}

Patient demographics are shown in $\bullet$ Table 1 . There were a total of 40 patients with IULAKT (the above and across stent groups: 17 and 23 patients, respectively). There were significant differences between the two groups pertaining to the average age $(P=$ 


\begin{tabular}{|llll}
\hline Factors & Above stent & Across stent & P value \\
\hline Number of patients & 17 & 23 & \\
\hline Age (years) & 67.5 & 72.5 & 0.0318 \\
\hline Sex (male/female; no./percent) & $10 / 7(58.8 / 41.2)$ & $17 / 6(73.9 / 26.1)$ & 0.0374 \\
\hline CA19-9 level (mg/dL) & 197.7 & 247.6 & 0.6818 \\
\hline Tumor type (nodular/sclerosing) [18] & $10 / 7(58.8 / 41.2)$ & $14 / 9(60.9 / 39.1)$ & 0.8894 \\
\hline Bithmuth type (III/IV) [19] & $7 / 10(41.2 / 58.8)$ & $14 / 9(60.9 / 39.1)$ & 0.0160 \\
\hline Artery invasion & $17 / 0(100 / 0)$ & $20 / 3(86.7 / 13.3)$ & 0.1279 \\
\hline Hilar lymph node metastasis & $10 / 7(58.8 / 41.2)$ & $15 / 8(65.2 / 34.8)$ & 0.6890 \\
\hline UICC stage (III/IV) [20] & $9 / 8(52.9 / 47.1)$ & $16 / 7(69.5 / 31.5)$ & 0.2950 \\
\hline
\end{tabular}

Table 1 Demographics of patients with Klatskin tumor.

Above stent: stent placed above the sphincter of Oddi

Across stent: stent placed across the sphincter of Oddi

Artery invasion: tumor invasion to the right and/or left hepatic artery

UICC, Union for International Cancer Control

\begin{tabular}{|llll|}
\hline Factors & Above stent & Across stent & P value \\
\hline Number of patients & $\mathbf{1 7}$ & $\mathbf{2 3}$ & \\
\hline Number of stents (1/2) & $8 / 9(47.1 / 52.9)$ & $19 / 4(82.6 / 17.4)$ & 0.0170 \\
\hline Stent to operation (days) & 139.4 & 141.6 & 0.8818 \\
\hline Post-procedure pancreatitis [17] & $2 / 15(11.8 / 88.2)$ & $1 / 22(4.3 / 95.7)$ & 0.3916 \\
\hline Emergency ERCP & $14 / 3(82.4 / 17.6)$ & $22 / 1(87.5 / 12.5)$ & 0.1743 \\
\hline 1) Stent occlusion & $6 / 11(35.3 / 64.7)$ & $13 / 11(56.5 / 48.5)$ & 0.6124 \\
\hline 2) Stent dislocation & $2 / 15(11.8 / 81.2)$ & $7 / 16(30.4 / 69.6)$ & 0.1704 \\
\hline 3) Reflux cholangitis & $2 / 15(11.8 / 81.2)$ & $5 / 23(21.7 / 78.3)$ & 0.424 \\
\hline 4) Undrained area cholangitis & $5 / 12(29.4 / 70.6)$ & $15 / 8(65.2 / 34.8)$ & 0.0250 \\
\hline Stent patency >90 days & $11 / 6(60.9 / 39.1)$ & $1 / 22(12.5 / 87.5)$ & $<0.0001$ \\
\hline Stent patency (days) & 99.8 & 31.0 & $<0.0001$ \\
\hline
\end{tabular}

Table 2 Stent placement for patients with Klatskin tumor.

Above stent: stent placed above the sphincter of Oddi

Across stent: stent placed across the sphincter of Oddi

ERCP, endoscopic retrograde cholangiopancreatography

(): \%

\begin{tabular}{|llll|}
\hline Factors & Above stent & Across stent & P value \\
\hline Number of patients & $\mathbf{1 7}$ & $\mathbf{2 3}$ & \\
\hline Resection & $8 / 9(47.1 / 52.9)$ & $16 / 7(69.6 / 30.4)$ & 0.1587 \\
\hline R0 surgery & $5 / 12(29.4 / 70.6)$ & $13 / 10(56.5 / 43.5)$ & 0.0928 \\
\hline Surgical time (Minutes) & 688.6 & 819.3 & 0.0759 \\
\hline Operative blood loss (mL) & 931.1 & 1121.7 & 0.1907 \\
\hline Length of hospital stay (days) & 22.6 & 39.5 & 0.0475 \\
\hline Prognosis (months) & 24.8 & 20.4 & 0.3277 \\
\hline
\end{tabular}

Table 3 Surgical results in patients with Klatskin tumor.

Above stent: stent placed above the sphincter of Oddi

Across stent: stent placed across the sphincter of Oddi ( ): \%

$0.0318)$ and sex distribution $(P=0.0374)$. The serum CA19- 9 level was higher in the across stent group than the above stent group. The distribution of nodular type was similar between the two groups. For Bismuth classification [19], type IV was significantly associated with the above stent group than the across stent group $(P=0.016)$. Artery involvement tended to be recognized in the above stent group compared with the across stent group. Regarding hilar lymph node metastasis, there were no significant differences between the two groups according to the UICC classification [20]. Therefore, more severe Klatskin tumors tended to be observed in the above stent group than the across stent group.

\section{Outcome of stent placement}

Data for endoscopic procedures, including emergency ERCP, are indicated in Table 2. Stent placement was successfully performed in all patients. For the number of stent placements, single stent placement was significantly more likely in the across stent group $(82.6 \%)$ than the above stent group $(47.1 \%)(P=0.0170)$.
We performed further evaluation of above stent placement. There were significant differences in median times (days) between stent insertion and each complication occurrence (above stent vs. across stent): 103 vs. 31.5 pertaining to stent occlusion, 134 vs. 42 pertaining to stent dislocation, 70 vs. 20.6 pertaining to reflux cholangitis, and 88.4 vs. 32.2 pertaining to undrained area cholangitis $(P<0.05)$. The median time from stent placement to surgery in the overall subject population was 139.4 days in the above stent group and 141.6 days in the across stent group.Stent dislocation tended to be higher in the across stent group (30.4\%) than the above stent group (11.8\%) $(P=0.1704)$. Developing undrained areas of cholangitis were significantly greater in the across stent group (65.2\%) than the above stent group (29.4\%) $(P=0.0250)$. Stent patency over 90 days was $60.9 \%$ in the above stent group and $12.5 \%$ in the across stent group $(P<0.0001)$. The median stent patency period was 99.8 days in the above stent group and 31.0 days in the across stent group $(P<0.0001)$. There were no significant differences regarding the incidence of post- 


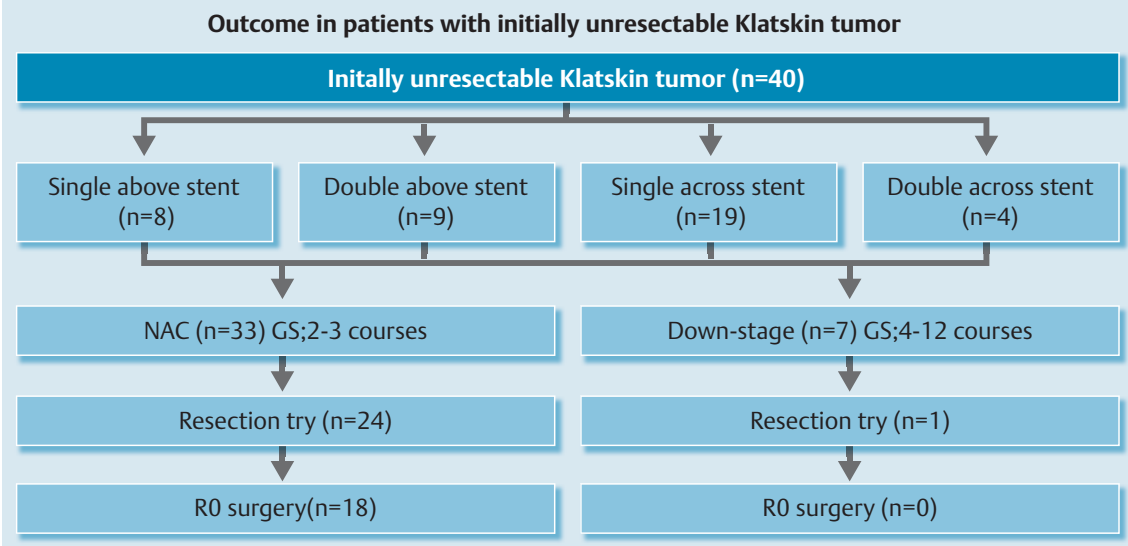

Fig. 5 Outcome in patients with Klatskin tumor. We tried neoadjuvant chemotherapy (NAC) for two or three courses, then continued down-staging of chemotherapy in 7 out of 40 patients. Finally, five patients with above stent and 13 patients with across stent were successfully treated with R0 surgery. This regimen (GS) provides patients with gemcitabine $1000 \mathrm{mg} / \mathrm{m}^{2}$ on days 1 and 8 plus $\mathrm{S}-1$ 60 , or $80 \mathrm{mg} /$ day on days 1 through 14 of a 21 -day cycle.

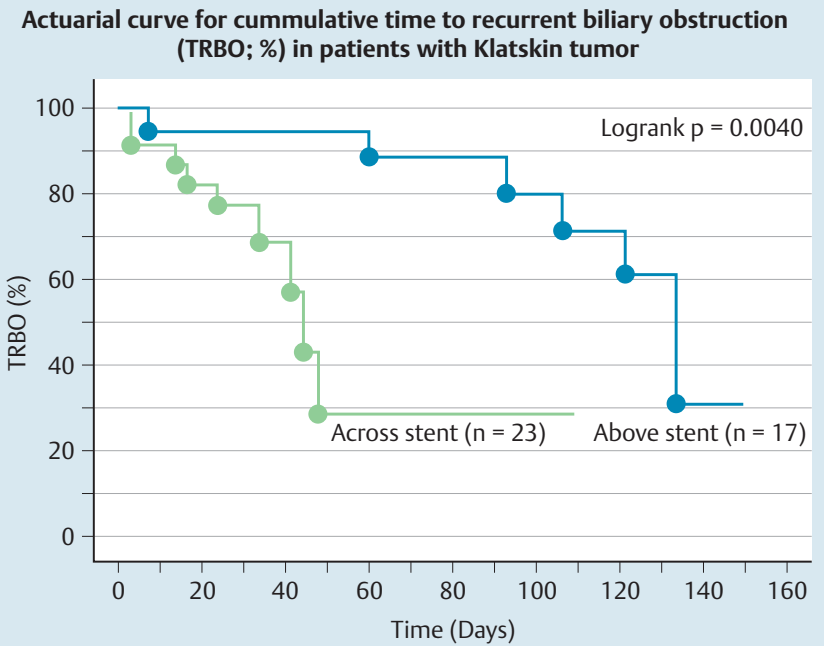

Fig. 6 Regarding NPR, above stent placement is superior to the group using across stent (logrank test, $P=0.0040$ ).

procedure pancreatitis or rates of emergency ERCP between the two groups.

\section{Surgical results}

- Table 3 shows the surgical results. Resection was successfully performed in the above stent $[47.1 \%(8 / 17)]$ and across stent [69.6\% (16/23)] groups. After performing NAC, we continued to down-stage chemotherapy in 7 of 40 patients ( 4 patients in the above stent group and 3 patients in the across stent group) ( $\bullet$ Fig.5). The rates of achievement of R0 resection in the above stent and across stent groups were 29.4\% (5/17) and 56.5\% (13/ 23 ), respectively. For the duration of hospital stay, it took 22.6 days for the above stent group and 39.5 days for the across stent group $(P=0.0475)$. There were no significant differences in the surgical time, blood loss, and prognosis between the two groups.

\section{Stent patency and multivariate analysis}

TRBO (stent patency-\%) on Kaplan-Meier curve is shown in - Fig. 6. TRBO for the first 30 days in the above stent and across stent groups was $94 \%$ and $77 \%$, respectively. TRBO for the next 30 days in the above stent and across stent groups was $88 \%$ and $29 \%$, respectively. TRBO for the next 30 days in the above stent and across stent groups were $80 \%$ and $29 \%$, respectively. Therefore, with respect to TRBO, the above stent group is superior to the across stent group (log rank test, $P=0.0040$ ). There were no sig-

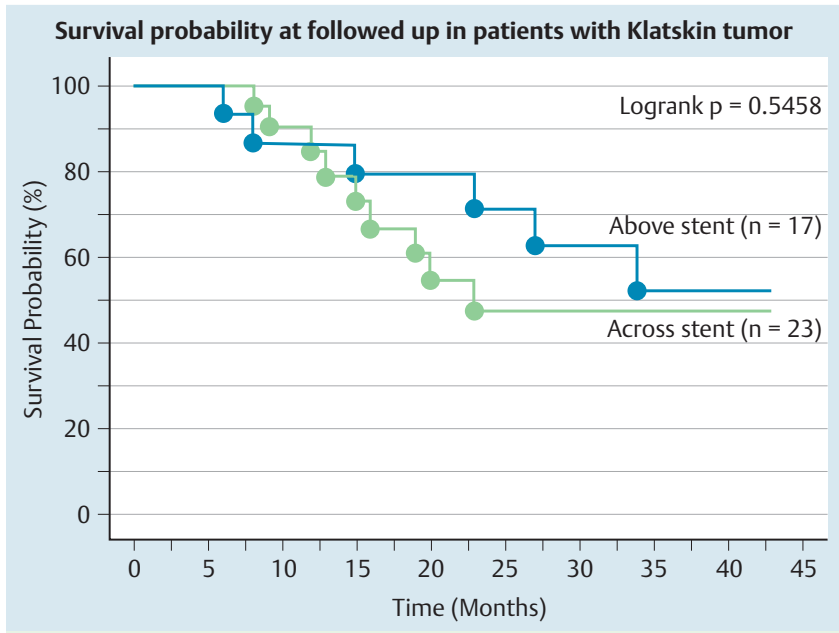

Fig. 7 Survival probability in patients with Klatskin tumor with above stent or across stent. There were no differences in survival between the two groups.

nificant differences in survival probability between the above stent and across stent groups ( $\bullet$ Fig. 7). Finally, we studied factors regarding stent patency over 90 days, which ensured safe NAC. The univariate analysis showed that presence of an undrained area of cholangitis tended to be lower in the group with stent patency over 90 days than in the counterpart $(P=0.0802)$ and above stent placement was significantly correlated in the group with stent patency over 90 days. Multivariate analysis using these factors revealed that above stent placement (odds ratio $=33.638, P=0.0048)$ was the only significant independent factor associated with stent patency over 90 days ( $\bullet$ Table 4 ).

Table 4 Multivariate analysis for stent patency $>90$ days.

\begin{tabular}{|llll}
\hline Factors & Hazard ratio & $\mathbf{9 5 \%} \mathbf{C l}$ & $\boldsymbol{P}$ value \\
\hline Age & 0.966 & $0.868-1.089$ & 0.5748 \\
\hline Sex & 1.119 & $0.179-6.984$ & 0.9038 \\
\hline Undrained area cholangitis & 0.559 & $0.085-3.668$ & 0.5446 \\
\hline Above stent placement & 33.638 & $2.922-387.303$ & 0.0048
\end{tabular}

Above stent: stent placed above the sphincter of Oddi $95 \% \mathrm{Cl}$ : 95\% confidence interval 


\section{Discussion}

$\nabla$

We examined above stent placement for patients with IULAKT undergoing NAC, which may lead to a successful R0 resection. This unmixed IULAKT group was selected and excluded intrahepatic cholangioma, metastatic tumor, gallbladder and biliary cancers arising from pancreatic head lesions. NAC for at least 3 months (90 days) is essential for safe control of biliary infection and jaundice. As our data indicated, plastic stent placement above the papilla could be an alternative to the conventional method for IULAKT.

NAC can provide patients who have initially unresectable cancer with the possibility of $\mathrm{RO}$ resection. Klatskin tumors result in symptomatic obstructive cholangitis, which sometimes hinders implementation of NAC. Any course of action must involve the safe and effective placement of a biliary stent during NAC without delay. With improvements in stenting, the management of Klatkin tumor merits a multidisciplinary approach, including NAC. Although biliary drainage is considered controversial for upfront surgery of Klatkin tumors [21,22], NAC has an important role as an adjunct to surgery for IULAKT [6]. Patient drainage of the obstructive biliary system is necessary before NAC is commenced. However, consensus and/or suitable standards for drainage methods using stent devices has been lacking in patients with unmixed Klatskin tumor.

Although the across stent device is a choice for obstructive jaundice of Klatskin tumor, the across stent device can induce reflux cholangitis and multiple across stent insertions are required for endoscopic sphincterotomy [7]. Cholangitis developed through the combination of reflux digestive juice and incomplete drainage of the biliary tract. In comparison with above stent devices, across stent devices may predispose patients to cholangitis because papillary function is more impaired with their use [23]. Our data also indicated that there were significant differences in median times (days) between stent insertion and the occurrence of each complication (above stent vs. across stent) in stent occlusion, stent dislocation, reflux cholangitis and undrained area of cholangitis $(P<0.05)$. Therefore, above stent placement rather than across stent placement could prolong the onset of stent issues such as stent occlusion, stent dislocation, reflux cholangitis, and undrained area of cholangitis.

In addition, across stent placement tended to result in stent dislocation rather than above stent placement, according to our data. Recently, ENBD placement was recommended in patients with Klatskin tumor [8]. However, the duration for patients may extend to less than 2 or 3 weeks and it may not always be useful because of patient tolerance for it. An expandable metallic stent is not suitable for resection because the line of resection is obscure and it may also result in adhesions to the tumor margin. Above stent placement is reported as being useful for reducting reflux cholangitis in patients with biliary strictures $[15,24]$. Our data reporting good stent patency corroborate these studies. Therefore, we propose the implementation of above stent devices for patients with IULAKT undergoing NAC.

Advances in NAC and surgical technology have improved the prognosis for patients with Klatskin tumor [6,25]. Although biliary tract cancers are sensitive to chemotherapy and GEM plus cisplatin confers survival advantage, this chemotherapy regimen can only extend life expectancy by a mere 3 months without surgery [26]. However, NAC may contribute to prolonged survival in patients with IULAKT. Because it takes more than 3 months to perform this procedure at our institute, we need a safe NAC peri- od without interruption. A concern pertaining to providing a safe NAC period is obstructive cholangitis because of stent occlusion during this period. Preoperative biliary stenting itself augments the risk of postoperative morbidity and prolonged hospital stay [27]. EST and/or conventional across stent placement can result in Oddi dysfunction [28]. Conversely, above stent placement is safe and feasible without the deterioration of duodenal papilla function. As our data indicated, above stent placement demonstrated longer stent patency compared with across stent placement and extended the TRBO, as shown in 0 Table 2 and - Fig.6. Across stent placement can be a risk for stent dysfunction because patients with across stent devices always suffer from excessive duodenal juice, which may cause reflux cholangitis [29]. Above stent placement can preserve the function of duodenal papilla. Endoscopic sphincterotomy for above stent placement becomes obsolete even if multiple stents are planned [15]. For the maintenance of the above stent device, there are sometimes difficulties in its retrieval; however, a string can be attached to this device to overcome this concern $[15,16,30]$. The above stent device can be easily removed and reduces the risk of cholangitis. However, once cholangitis has occurred, it can be refractory and may impede safe NAC and resection. Therefore, we recommend using the above stent device for first-line stent placement. Because dislocation of the above stent device is observed in patients with IULAKT, we preserve one or two flaps at the hilar direction to prevent dislocation. Furthermore, the across stent device is more likely to be nudged out of the collecting position and dislocated from the proper position because it is attached at two points, one in the ampulla and the other in the affected tumor lesion. These two points can move independently, which could induce across stent device dislocation.

For resection, stent placement for patients with IULAKT undergoing NAC affects surgical results such as surgical time, complication after surgery, and hospital stay. Table 3 shows that above stent placement was associated with a short hospital stay. Survival data for the above stent group failed to show superiority compared with the across stent group and there were no differences in the rate of R0 resection between the two groups. However, shorter surgical time and lower operative blood loss tended to be recognized in the above stent group. Multivariate analysis for stent patency over 90 days showed that above stent placement was the only independent factor for this outcome. Based on these results, above stent placement could be a better choice than across stent placement with respect to surgical merits.

This study has several limitations. Our data were retrospective, and the study was conducted in a single center as a cohort study. In addition, the above stent device, $7 \mathrm{~F}$ in diameter, showed short stent patency. However, multiple above stent devices, $7 \mathrm{~F}$ in diameter, could be feasible without sphincterotomy. Although larger-diameter stents, including those with diameters over $8 \mathrm{~F}$, may prolong stent patency, sphincterotomy-induced reflux cholangitis was mandatory and their planned retrieval was difficult. Moreover, larger-diameter stents would enhance the risk of post-procedure pancreatitis. TRBO for the above stent device was reported as being over 130 days $[9,30]$. Our data showed that stent patency was 31 days in the across stent group compared with 99 days in the above stent group. NAC itself may induce bile duct infection by its immunosuppressive effect and shorten TRBO.

In conclusion, above stent placement is an appropriate option for the stenting of patients with symptomatic IULAKT, who are candidates for NAC, and where there is more than the minimum 3 
months to control cholangitis. Unilateral above stent placement should initially be attempted. If it is insufficient in controlling jaundice, bilateral stents should be placed. Above stent placement could minimize reintervention in recurrent biliary obstruction for IULAKT. Additional benefits include less opportunity for stent dislocation and a potentially shortened hospital stay that promotes reduced cost, when above stent placement is compared with the conventional across stent placement.

\section{Competing interests: None}

\section{References}

1 Jamagin WR, Fong $Y$, Metatteo RP et al. Staging, Resectability, and outcome in 225 patients with hilar cholangiocarcinoma. Ann Surg 2001; 234: $507-519$

2 Finn MP, Annmarie TL, Ove BSM et al. Randomized trial of stent placed above and across the sphincter of Oddi in malignant bile duct obstruction. Gastrointest Endosc 1998; 48: 574-582

3 Miyakawa S, Ishihara S, Horiguchi A et al. Biliary tract cancer treatment: 5548 results from the biliary tract cancer statistics registry from 1998 to 2004 in Japan. J Hepatobiliary Pancreas Science 2009; 16: $1-7$

4 Ruys AT, van Beem BE, Engelbrecht MR et al. Radiological staging in patients with hilar cholangiocarcinoma: A systemic review and meta-analysis. Br J. Radiol 2012; 85: 1255-1262

$5 \mathrm{Kim} \mathrm{SH}, \mathrm{Kim}$ IH, Kim SW. Repetitive response to gemcitabine that led to curative resection in cholangiocarcinoma. World J Gastroenterol 2009; 15: $4593-4595$

6 Kato A, Shimizu H, Ohtsuka $M$ et al. Surgical resection after downstaging chemotherapy for initially unresectable locally advanced biliary tract cancer: A retrospective single-center study. Ann Surg Oncol 2013; 20: 318-324

7 Weickert $U$, Venzke T, Konig J et al. Why do bilioduedenal plastic stents become occuluded? A clinical and pathological investigation on 100 consecutive patients Endoscopy 2001; 33: 786 - 790

8 Kawakami H, Kuwatani M, Onodera $M$ et al. Endoscopic nasobiliary drainage is the most suitable preoperative biliary drainage method in the management of patients with hilar cholangiocarcinoma. J Gastroenterol 2011; 46: $242-248$

9 Liu $Q$ Khay G, Cotton PB. Feasibility of stent placement above the sphincter of Oddi ("inside-stent") for patients with malignant biliary obstruction. Endoscopy 1998; 30: 687-690

10 Pedersen FM, Lassen AT, Schaffalitzky de Muckadell OB. Randomized trial of stent placed above and 11across the sphincter of Oddi in malignant bile duct obstruction. Gastrointest Endosc 1998; 48: 574- 579

11 Isayama H, Hamada T, Yasuda I et al. The Tokyo criteria 2014 for transpapillary biliary biliary stenting. Digestive Endosc 2015; 27: 259-264

12 Endo I, Shimada H, Sugita $M$ et al. Role of three dimensional imaging in operative planning for hilar cholangiocarcinoma. Surgery 2007; 142: $666-675$
13 Eisenhaiser EA, Therasse P, Bogaerts J et al. New response evaluation criteria in solid tumors: Revised RECIST guideline (version 1.1). Eur J Cancer 2009; 45: 228-247

$14 \mathrm{Seo} \mathrm{H}$, Lee JM, Kim IH et al. Evaluation of the gross type and longitudinal extent of extrahepatic cholangiocarcinomas on contrast-enhancement multidetector row computed tomography. J Comput Assist Tomogr 2009; 33: $376-382$

15 Ishiwatari $H$, Hayashi $T$, Ono $M$ et al. Newly designed plastic stent for endoscopic placement above the sphincter of Oddi in patients with malignant hilar biliary obstruction. Dig Endosc 2013; 25: 94-99

16 Kobayashi $N$, Watanabe $S$, Hosono $K$ et al. Endoscopic inside stent placement is a suitable as a bridging treatment for preoperative biliary tract cancer. BMC Gastroenterol 2015; 15: 8

17 Cotton PB, Lehman G, Vennes J et al. Endoscopic sphincterotomy complication and their management: an attempt at concensus. Gastrointest Endosc 1991; 37: 383-393

18 Weinbren K, Mutum SS. Pathological aspects of cholangiocarcinoma. J Pathology 1983; 139: 217-238

19 Bithmuth $H$, Nakache R, Diamond T. Management strategies in resection for hilar biliary cholangiocarcinoma. Ann Ssurg 1992; 215: 31 - 38

20 Juntermanns B, Sotiropoulos GC, Radunz $S$ et al. Comparison of the sixth and the seventh editions of the UICC classification for perihilar cholangiocarcinoma. Ann Surg Oncol 2013; 20: 277-284

21 Iacono C, Ruzzenente A, Campagnaro T et al. Role of preoperative biliary drainage in jaundiced patients who are candidates for pancreatoduodenectomy or hepatic resection: highlights and drawbacks. Ann Surg 2013; 257: 191 - 204

22 Saxena P, Kumbhari V, Zein ME et al. Preoperative biliary drainage. Dig Endosc 2015; 27: 265-277

23 Okamoto T, Fujioka S, Yanagisawa S et al. Placement of a metallic stent across the main duodenal papilla may predispose to cholangitis. Gastrointest Endosc 2006; 63: 792 -796

24 Hisatsune H, Yazumi S, Egawa $\mathrm{H}$ et al. Endoscopic management of biliary strictures after duct-to-duct biliary reconstruction in right-lobe living-donor liver transplantation. Transplantation 2003; 765: 810815

25 Shimizu H, Kimura F, Yoshitomi $H$ et al. Aggressive surgical resection of hilar cholangiocarcinoma of the left-side predominance: radicality and safety of left-sided hepatectomy. Ann Surg 2010; 251: 281 - 286

26 Valle J, Wasan H, Palmer DH et al. Cisplatin plus gemcitabine for biliary tract cancer. N Engl J Med 2010; 362: $1273-1281$

27 Kloek JJ, Heger M, van der Gaag NA et al. Effect of preoperative biliary drainage on coagulation and fibrinolysis in severe obstructive cholangitis. J Clin Gastroenterol 2010; 44: 646-652

28 Yasuda I, Tomita E, Enya $M$ et al. Can endoscopic papillary baloon dilation really preserve sphincter of Oddi function? Gut 2001; 49: 686691

29 Leung JW, Liu YL, Desta T et al. Is there a synergistic effect between mixed bacterial infection in biofilm formation on biliary stents? Gastrointest Endosc 1998; 48: 250-257

30 Inatomi $\mathrm{O}$, Bamba S, Shioya $\mathrm{N}$ et al. Threaded biliary inside stents are a safe and effective therapeutic option in cases of malignant hilar obstruction. BMC Gastroenterol 2013; 13: 31 